\title{
Curcumin liposomes prepared with milk fat globule membrane phospholipids and soybean lecithin
}

\author{
Hong-Hao Jin, ${ }^{* 1}$ Qun Lu, ${ }^{*}{ }^{1}$ and Jian-Guo Jiang ${ }^{* 2}$ \\ ${ }^{*}$ College of Food and Bioengineering, South China University of Technology, Guangzhou, 510640, China \\ †College of Food Science and Technology, Huazhong Agricultural University, Wuhan 470070, China
}

\begin{abstract}
Using thin film ultrasonic dispersion method, the curcumin liposomes were prepared with milk fat globule membrane (MFGM) phospholipids and soybean lecithins, respectively, to compare the characteristics and stability of the 2 curcumin liposomes. The processing parameters of curcumin liposomes were investigated to evaluate their effects on the encapsulation efficiency. Curcumin liposomes were characterized in terms of size distribution, $\zeta$-potential, and in vitro release behavior, and then their storage stability under various conditions was evaluated. The curcumin liposomes prepared with MFGM phospholipids had an encapsulation efficiency of about $74 \%$, an average particle size of 212.3 $\mathrm{nm}$, and a $\zeta$-potential of $-48.60 \mathrm{mV}$. The MFGM liposomes showed higher encapsulation efficiency, smaller particle size, higher absolute value of $\zeta$-potential, and slower in vitro release than soybean liposomes. The retention rate of liposomal curcumin was significantly higher than that of free curcumin. The stability of the 2 liposomes under different $\mathrm{pH}$ was almost the same, but MFGM liposomes displayed a slightly higher stability than soybean liposomes under the conditions of $\mathrm{Fe}^{3+}$, light, temperature, oxygen, and relative humidity. In conclusion, MFGM phospholipids have potential advantages in the manufacture of curcumin liposomes used in food systems.
\end{abstract}

Key words: curcumin, liposome, milk fat globule membrane, phospholipid, soybean lecithin

\section{INTRODUCTION}

Curcumin, one of the phenolic compounds derived from turmeric, is commonly used as a spice or food colorant. Curcumin has been shown a variety of bio-

Received September 13, 2015.

Accepted December 3, 2015.

${ }^{1}$ These authors contributed to the work equally.

${ }^{2}$ Corresponding author: jgjiang@scut.edu.cn logical activities, such as antitumor, antioxidant, antiinflammatory functions, and the capacity of lowering blood pressure (Khar et al., 1999; Anand et al., 2007, 2008). However, curcumin is susceptible to basic $\mathrm{pH}$, light, and temperature, which reduce its stability. The poor chemical stability and low bioavailability of curcumin limit its applications in food and pharmaceutical industries.

The preparation of curcumin liposomes can protect curcumin from unfavorable conditions. Liposomes, spherical bilayer vesicles, are amphipathic phospholipid bilayer membranes with an aqueous core inside and hydrophobic layer on the surface. Liposomes have been demonstrated to possess many typical advantages including biodegradability, biocompatibility, solubilization of poorly soluble compounds, and sustained-releasing capability (Kima and Baianua, 1991; Neethirajan and Jayas, 2011). In the food industry, liposome is often used to encapsulate nutraceuticals, food flavors, food colorants, and food antimicrobials (Keller, 2001; Taylor et al., 2005). The traditional liposomal materials are highly purified lecithin extracted from soybean or egg yolk. The lipid systems presented the benefit of low toxicity compared with polymeric materials.

Phospholipids are one of the main constituents of milk fat globule membranes (MFGM). The nutritional and functional importance of MFGM-derived phospholipids has attracted more and more researchers to apply it in their studies (Spitsberg, 2005; Dewettinck et al., 2008). The MFGM phospholipids not only contain phosphatidylcholine, phosphatidylethanolamine, and phosphatidylinositol like those in soybean phospholipids, but also have a high level of sphingomyelin, which has been used as an alternative source of soybean phospholipids for food supplements (Astaire et al., 2003). On the other hand, MFGM phospholipids tend to be more saturated than soybean phospholipids because of the presence of a higher degree of saturation of fatty acid chains in the MFGM phospholipid molecules. Studies have shown that MFGM phospholipids can also be employed to prepare liposomes (Thompson et al., 2006; Thompson and Singh, 2006; Thompson et al., 2007). Compared with conventional soybean-derived materials, MFGM 
phospholipids, with unique compositions and lower cost, may have broader application prospects.

Some studies have reported curcumin liposomes encapsulated by traditional or synthetic lecithin (Began et al., 1999; Takahashi et al., 2009; Patra et al., 2013), but the curcumin liposomes prepared with MFGM phospholipids have not been investigated. The objectives of the present study, respectively preparing curcumin liposomes with MFGM phospholipids and soybean lecithin, were to examine the ability of MFGM phospholipids to encapsulate curcumin by comparing the characteristics and stability of MFGM liposomes with those prepared with soybean lecithin. The processing parameters, size distribution, $\zeta$-potential, and in vitro release of the 2 liposomes were investigated and determined. Their stability under various conditions, including $\mathrm{pH}$, metal irons, light, temperature, oxygen, and relative humidity, was evaluated.

\section{MATERIALS AND METHODS}

\section{Materials and Apparatus}

The following materials were used in this study: curcumin (Aladdin Industrial Co., Ltd., Shanghai, China), MFGM phospholipids (prepared and preserved by our laboratory), soybean lecithin (Aladdin Industrial Co., Ltd., Shanghai, China), UV-Vis spectrophotometer [UV-2102PC, UNICO (Shanghai) Instruments Co., Ltd., China], Zetasizer Nano ZS (Malvern Instruments Co., Ltd., Malvern, UK), and $\mathrm{pH}$ meter (S-3D, Shanghai Precision and Scientific Instrument Co., Ltd., Shanghai City, China).

\section{Preparation of Curcumin Liposomes}

To compare the MFGM phospholipids with traditional membrane materials, the curcumin liposomes were prepared with MFGM phospholipids and soybean lecithin, respectively. The thin film ultrasonic dispersion method, a general procedure to obtain MFGM (Vermette et al., 2002) from fresh buffalo milk, was performed to prepare the liposomes. The MFGM phospholipids/soybean lecithin was dispersed in chloroform. Curcumin was dissolved in anhydrous ethanol and thoroughly mixed with the MFGM phospholipids/soybean lecithin dispersion in a $250-\mathrm{mL}$ round-bottomed flask. The mixture solution was evaporated on a rotary evaporator at $35^{\circ} \mathrm{C}$, until a thin film was formed on the flask. The film was washed with PBS and completely hydrated for $2 \mathrm{~h}$. After the hydration of thin lipid film with PBS, the lipid dispersion was sonicated for $4 \mathrm{~min}$, and finally the liposomes encapsulated with curcumin were obtained.

\section{Determination of Curcumin Content and Encapsulation Efficiency}

Precisely weighed curcumin was dissolved and diluted with anhydrous ethanol to form $0.4,0.6,0.8,1.0,1.2$, $1.4,1.6,2.0,2.4,2.8,3.2 \mu \mathrm{g} / \mathrm{mL}$ of standard solution, the absorbance of which was measured at $424 \mathrm{~nm}$ with anhydrous ethanol as the blank. The linear regression analysis of absorbance on concentration was conducted and the regression equation was obtained. The regression equation of the standard curve was $\mathrm{y}=0.1718 \mathrm{x}$ $+0.003, R^{2}=0.999$. Every sample was diluted with anhydrous ethanol to a certain concentration, measured its absorption at $424 \mathrm{~nm}$, and calculated the curcumin content according to the regression equation.

Encapsulation efficiency is a significant indicator in the quality evaluation of liposomes (Xia et al., 2012). The encapsulation efficiency (EE) was determined as follows:

$$
\mathrm{EE}=\frac{m_{1}-m_{2}}{m_{1}} \times 100 \%,
$$

where $\mathrm{m}_{1}$ is the amount of total curcumin and $\mathrm{m}_{2}$ is the amount of free curcumin in the liposome solution. Separation of liposome-incorporated and free curcumin was accomplished by centrifugation at $15,000 \times g$ for $30 \min$ at $4^{\circ} \mathrm{C}$.

\section{Investigation of Processing Parameters}

The assessment of processing parameters was performed to analyze their influences on the encapsulation efficiency and determine the optimal conditions for preparing curcumin liposomes. The effects of ratio of curcumin to phospholipids/lecithin, ultrasonic time, PBS pH, concentration of PBS, and volume of PBS on the encapsulation efficiency were investigated in this study. The individual conditions of the 5 parameters were carried out as follows: ratio of curcumin to MFGM phospholipids 1:30, 1:35, 1:40, 1:45, 1:50; ratio of curcumin to soybean lecithin 1:10, 1:15, 1:20, 1:25, 1:30; ultrasonic time 1, 2, 3, 4, 5 min; PBS pH 5.0, 5.5, 6.0, 6.5, 7.0; concentration of PBS 0.005, 0.010, 0.020, $0.040,0.060 \mathrm{~mol} / \mathrm{L}$; and volume of PBS 10, 15, 20, 25, $30 \mathrm{~mL}$.

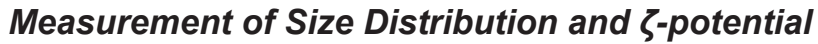

Prior to the measurement of size distribution and $\zeta$-potential, the liposome dispersions were diluted to the required turbidity with PBS solution. The average size and size distribution of curcumin liposomes were 
measured by dynamic light scattering using a Zetasizer Nano ZS (Malvern Instruments Co., Ltd.). Samples were analyzed at $25^{\circ} \mathrm{C}$ with a scattering angle of $90^{\circ}$. The $\zeta$-potential of curcumin liposomes was determined at $25^{\circ} \mathrm{C}$ using laser Doppler microelectrophoresis on a Zetasizer Nano ZS (Malvern Instruments Co., Ltd.).

\section{In Vitro Release of Curcumin from Liposomes}

This experiment was conducted using $10 \mathrm{~mL}$ of curcumin liposome dispersions dialyzed against $200 \mathrm{~mL}$ of PBS solution with stirring at $10 \mathrm{rpm}$ in a water bath at a temperature of $37^{\circ} \mathrm{C}$. The release of curcumin from the liposomal interiors was monitored as a function of time. Ten milliliters of external PBS solution was sampled, and an equal volume of PBS solution was replaced at the same time. The amount of curcumin in the samples was determined, and then the in vitro release profiles of curcumin liposomes were obtained. The dialysis behavior of free curcumin solution was also detected to make a comparison with that of curcumin liposomes. The formula to calculate cumulative release rate is

$$
\mathrm{Q}=\frac{\mathrm{M}_{\mathrm{n}}}{\mathrm{M}_{0}} \times 100 \%,
$$

where $Q$ is the cumulative release rate, $M_{n}$ is the amount of curcumin released from the liposomes at a given time, and $\mathrm{M}_{0}$ is the amount of curcumin initially encapsulated in the liposomes.

\section{Determination of Curcumin Retention Rate}

In the course of storage, curcumin was subject to various environmental factors such as light, high temperature, and oxygen, which led to the destruction and mass loss of curcumin. The retention rate (RR) of curcumin is an effective index for the assessment of stability of curcumin liposomes. The RR can be calculated by the following formula:

$$
\mathrm{RR}=\frac{\mathrm{C}_{\mathrm{A}}}{\mathrm{C}_{\mathrm{B}}} \times 100 \%,
$$

where $\mathrm{C}_{\mathrm{A}}$ and $\mathrm{C}_{\mathrm{B}}$ are the amount of curcumin existing in samples after and before a time of storage, respectively.

\section{pH Treatment on Curcumin Liposomes}

The solution of $\mathrm{pH}$ value $2,3,4,5,6,7,7.5,8,9$ was prepared. This solution was mixed 1:1 (vol/vol) with curcumin liposome dispersion, the curcumin amount of which was calculated through the measurement of adsorption at $424 \mathrm{~nm}$ by UV-vis spectroscopy [UNICO (Shanghai) Instruments Co., Ltd.]. The RR of encapsulated and unencapsulated curcumin was determined 30 min after the $\mathrm{pH}$ treatment and again 20 and $40 \mathrm{~h}$ later.

\section{Metal Ion Treatment on Curcumin Liposomes}

A mixture composed of curcumin liposome dispersions and metal ion solution at a ratio of $1: 1$ ( $\mathrm{vol} /$ vol) was used for this test. The concentration of metal ion solution was $0.01 \mathrm{~mol} / \mathrm{L}$, including $\mathrm{CaCl}_{2}, \mathrm{CuCl}_{2}$, $\mathrm{MgCl}_{2}, \mathrm{ZnCl}_{2}, \mathrm{NaCl}$, and $\mathrm{FeCl}_{3}$ solution. The mixture was left for 10 min before determining the RR of curcumin.

\section{Light Treatment on Curcumin Liposomes}

The stability of curcumin liposomes stored under light and in the dark was evaluated over a 30 -d period. Samples exposed to light were placed in a transparent jar, and other equivalent samples kept in a dark place were preserved in brown jars. The liposome dispersions were assessed every $3 \mathrm{~d}$ by measuring changes in RR.

\section{Temperature Treatment on Curcumin Liposomes}

Samples were held at 4,25 , and $50^{\circ} \mathrm{C}$, respectively, and protected from light. Curcumin liposome dispersions were sampled every $3 \mathrm{~d}$ for the examination of RR. The experimental period was $30 \mathrm{~d}$.

\section{Oxygen Treatment on Curcumin Liposomes}

Curcumin liposome dispersions were stored with and without oxygen, respectively, kept in the dark at room temperature. The remaining curcumin and liposomes were sampled to determine curcumin amount and determine their RR every $3 \mathrm{~d}$ in the consequent $30 \mathrm{~d}$.

\section{Relative Humidity Treatment on Curcumin Liposomes}

To estimate the effects of different relative humidity on the stability of curcumin liposomes during storage, samples were subjected to the following experimental treatments. A set of curcumin liposome dispersions was incubated with saturated magnesium chloride solution (relative humidity $33 \%$ ) for $30 \mathrm{~d}$, whereas another set of curcumin liposome dispersions was incubated with saturated potassium iodide solution (relative humidity 
$80 \%$ ) for $30 \mathrm{~d}$. Samples were taken every $3 \mathrm{~d}$ to examine curcumin amount for the calculation of $\mathrm{RR}$.

\section{RESULTS AND DISCUSSION}

\section{Analysis of MFGM and Phospholipids}

The MFGM contain 25 to $60 \%$ protein, whereas these proteins in other milk protein content are very little; MFGM protein accounted for 1 to $2 \%$ of total protein in milk (Mather, 2000). The SDS-PAGE of isolates in this research showed 6 to 8 major bands (Figure 1): mucins, xanthine dehydrogenase; periodate Schiff dilutes III-VII, butyrophilin, and caseins, which are similar to previous studies (Campagna et al., 2001; Ye et al., 2002). In addition to protein, other major components of MFGM are lipids, accounting for 25 to $45 \%$ (Rombaut et al., 2005). We obtained $0.87 \mathrm{~g}$ of lipid from $3 \mathrm{~g}$ of MFGM; MFGM lipid content in fresh buffalo milk was $29 \%$.

\section{Effects of Processing Parameters on the Encapsulation Efficiency of Curcumin Liposomes}

The encapsulation efficiency of liposomes progressively increased with the decrease of ratio of curcumin to phospholipids/lecithin (Figures 2A and 2B). The increased entrapment capacity of liposomes with increasing curcumin amount may indicate that part of the encapsulated curcumin is inserted or immobilized on the lipid membrane. But when the ratio further decreased, the encapsulation efficiency decreased, which can be explained as the amount of encapsulated curcumin in liposomes is dependent on the capacity of bilayers to accommodate it. The optimal ratio of curcumin to MFGM phospholipids and curcumin to soybean lecithin was 1:40 and 1:20, respectively, illustrating that different liposome membranes determined different optimal ratio.

The encapsulation efficiency of curcumin liposomes, prepared with MFGM phospholipids and soybean lecithin, reached the maximum when the ultrasonic time was 3 min (Figure 2C). Ultrasonic vibration has been commonly used in the preparation of unilamellar liposome vesicles. It has been reported that liposomes prepared with extensive sonication were quite clear, transparent, and stable for at least $30 \mathrm{~d}$ (Kim et al., 2007). On the other hand, excessive ultrasonic stress leads to the disintegration of liposomes. The ability of liposomes against external stress depends on the lipid composition, vesicle size, and surface charge (Yotsuyanagi et al., 1987). The incorporation of cholesterol into

\section{$\mathrm{kDa}$}

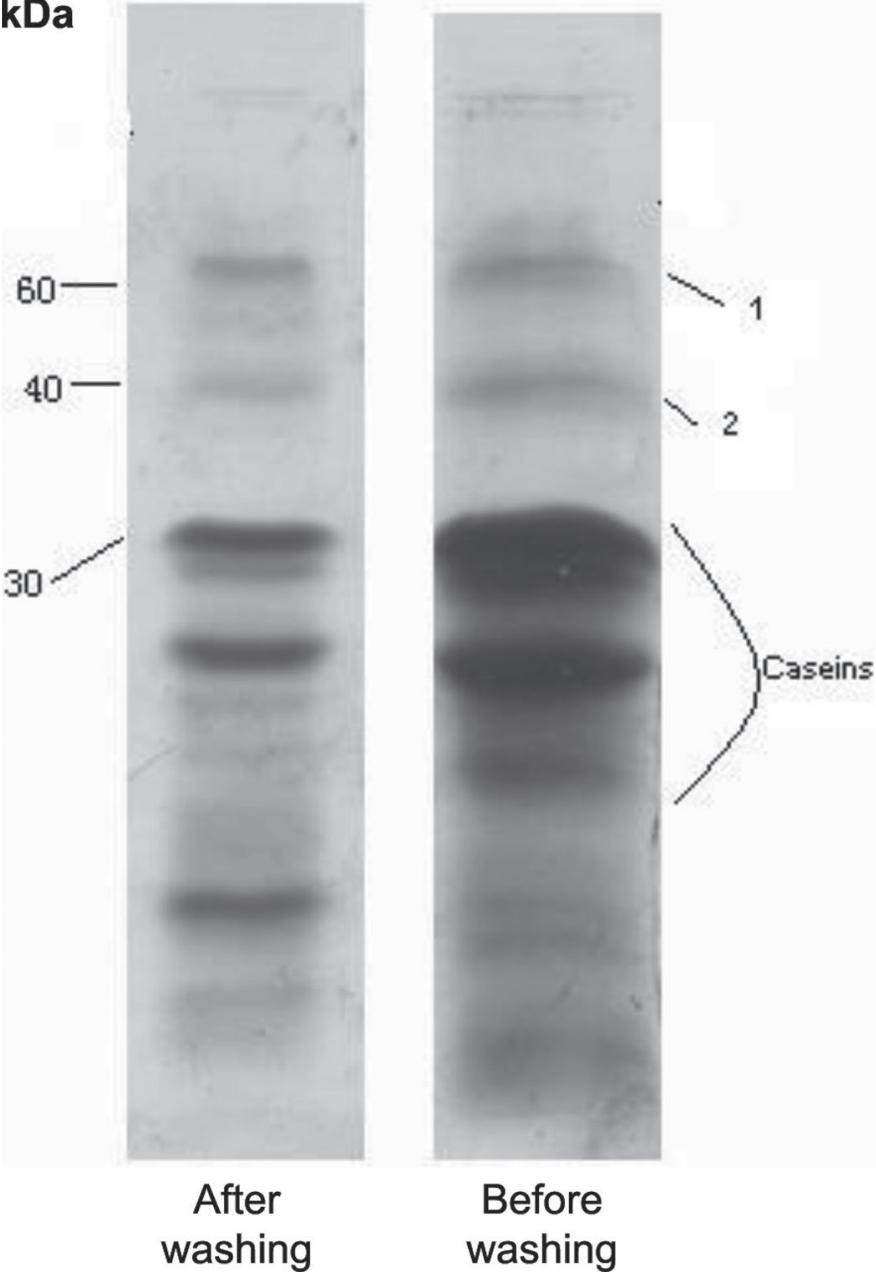

Figure 1. Polyacrylamide gel electrophoresis of milk fat globule membrane separated from fresh milk fat before and after washing. $1=$ butyrophilin; 2 = periodate Schiff dilute VII

the lipid composition of liposome membrane enhanced the membrane stability against ultrasonic stress ( $\mathrm{Lu}$ et al., 2011).

The encapsulation efficiency of liposomes prepared with MFGM phospholipids reached the peak at $\mathrm{pH}$ 5.5, whereas the optimal $\mathrm{pH}$ for soybean lecithin membrane was 6.0 (Figure 2D). Chemical stability of phospholipids has been considered in 2 parts: oxidation and hydrolysis (Grit and Crommelin, 1993). The hydrolysis of phospholipids is mainly affected by acid-base catalysis. Unsaturated fatty acid chains of phospholipids molecules are easy to be oxidized and hydrolyzed, and the productions of hydrolysis lower the $\mathrm{pH}$, accelerating the hydrolysis of liposomes. The oxidation and hydrolysis of phospholipids improve membrane permeability, resulting in the release of curcumin and decline of encapsulation efficiency. The MFGM phospholipids contain a 
higher degree of saturation of the fatty acid chains than soybean lecithin, which may bring a higher stability to the MFGM liposomes (Thompson et al., 2006).

A

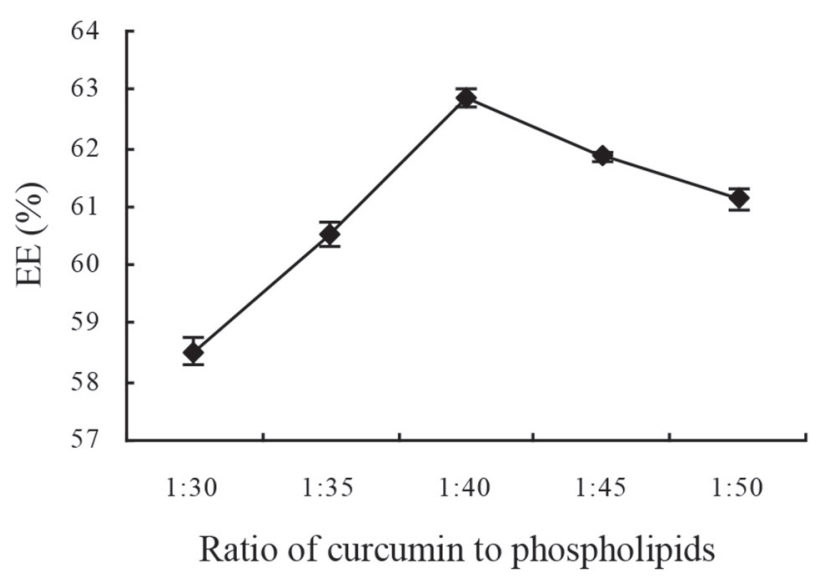

C

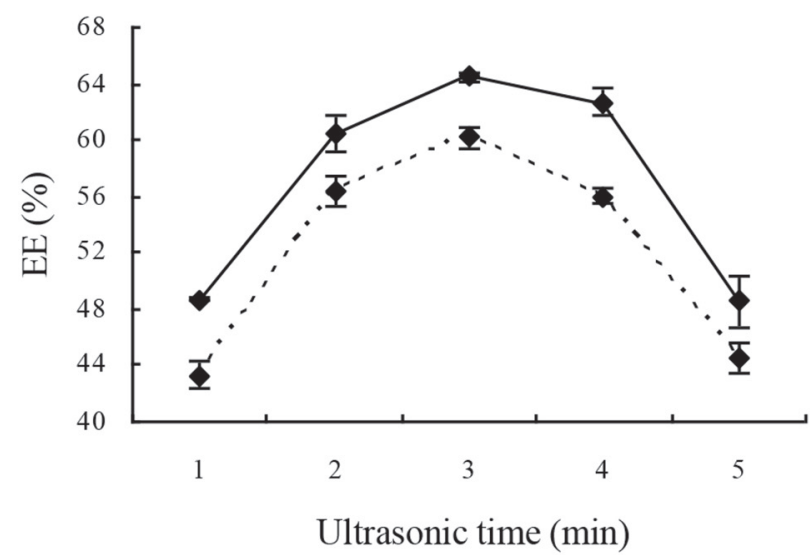

$\mathrm{E}$

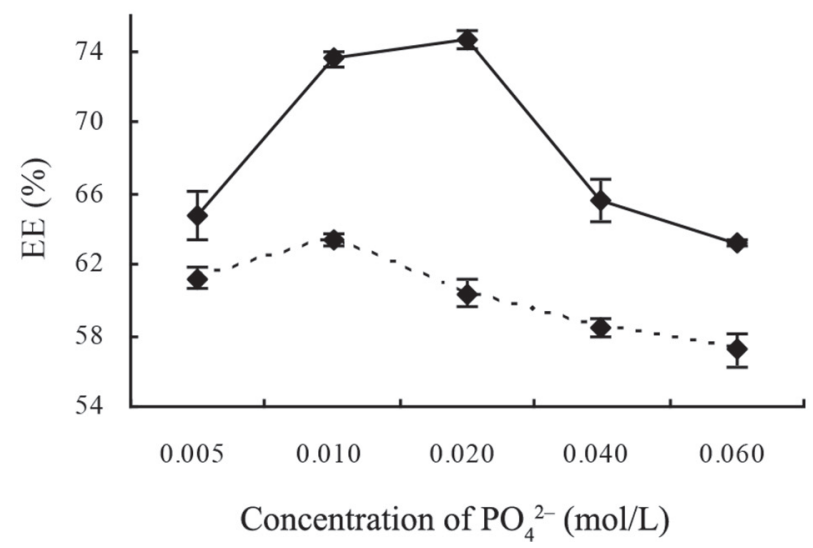

The best concentration $\left(\mathrm{PO}_{4}{ }^{2-}\right)$ and volume of PBS for curcumin liposomes prepared with MFGM phospholipids were $0.020 \mathrm{~mol} / \mathrm{L}$ and $15 \mathrm{~mL}$, respectively. The

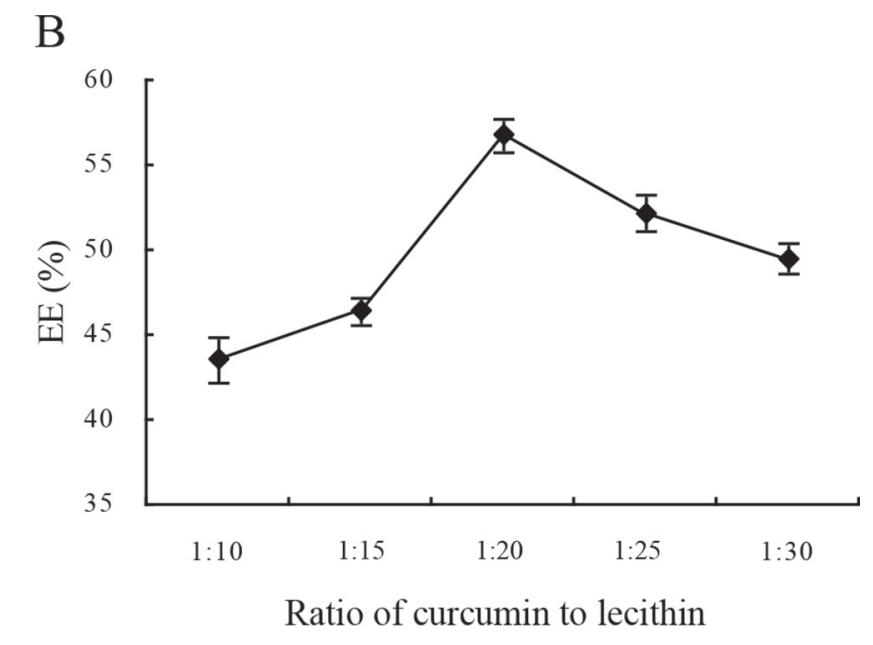

$\mathrm{D}$

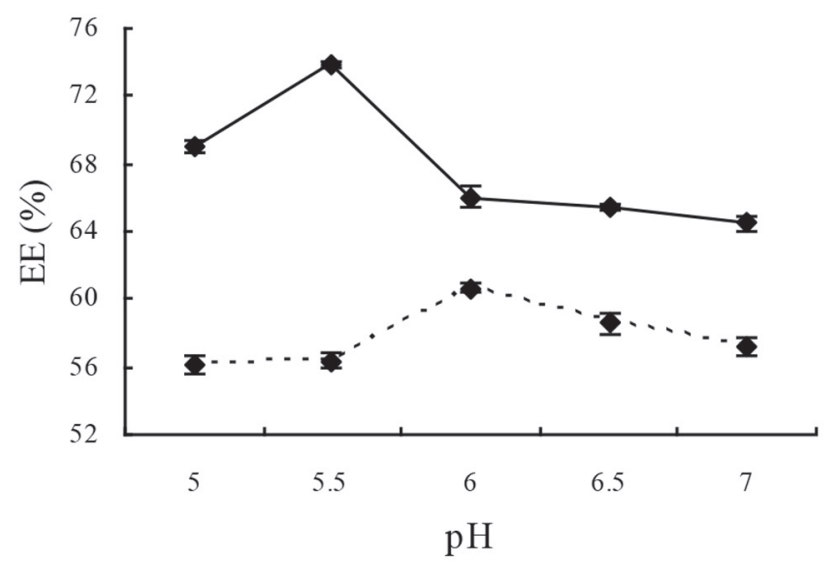

F

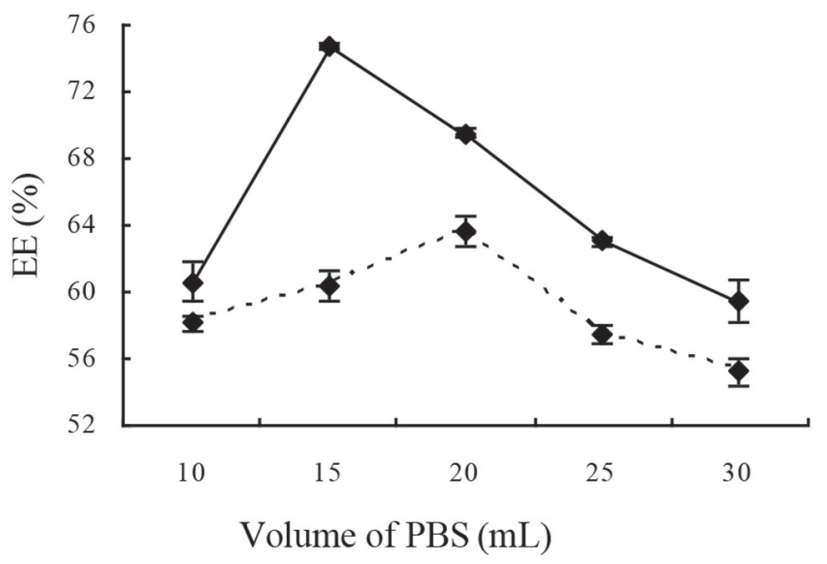

Figure 2. Effects of processing parameters on the encapsulation efficiency (EE) of curcumin liposomes prepared with either milk fat globule membrane (MFGM) phospholipids (-) or soybean lecithin (- - ). (A) Ratio of curcumin to MFGM phospholipids; (B) ratio of curcumin to soybean lecithin; (C) ultrasonic time; (D) PBS pH; (E) concentration of PBS $\left(\mathrm{PO}_{4}{ }^{2-}\right)$; (F) volume of PBS (n = 3, error bars indicate SD). 
encapsulation efficiency of curcumin liposomes prepared with soybean lecithin reached the highest when the concentration and volume of PBS was $0.010 \mathrm{~mol} / \mathrm{L}$ and $20 \mathrm{~mL}$, respectively (Figures $2 \mathrm{E}$ and $2 \mathrm{~F}$ ). Ionic strength and dilutions have no obvious effect on the encapsulation efficiency of liposomes compared with the above parameters, but immoderate concentration and volume of PBS was found to adversely influence the encapsulation efficiency. The effect of ionic strength on the physical stability of liposomes is in connection with the surface charge of liposomes (Crommelin, 1984).

The optimal conditions for curcumin liposomes prepared with MFGM phospholipids are ratio of curcumin to phospholipids 1:40, ultrasonic time $3 \mathrm{~min}, \mathrm{PBS} \mathrm{pH}$ 5.5 , concentration of PBS $0.020 \mathrm{~mol} / \mathrm{L}$, volume of PBS $15 \mathrm{~mL}$, whereas the optimal conditions for curcumin liposomes prepared with soybean lecithin are ratio of curcumin to lecithin 1:20, ultrasonic time 3 min, PBS $\mathrm{pH}$ 6.0, concentration of PBS $0.010 \mathrm{~mol} / \mathrm{L}$, volume of PBS $20 \mathrm{~mL}$. The amount of curcumin encapsulated was measured immediately following liposome preparation. All the following experiments on the encapsulated liposomes were then conducted on curcumin liposome dispersions prepared with MFGM phospholipids/soybean lecithin under optimal conditions. The encapsulation efficiency of the 2 curcumin liposomes was about 74 and $63 \%$, respectively. The MFGM liposomes showed higher load capacity probably because MFGM phospholipids possess more suitable phospholipids compositions and structures for curcumin liposome preparation, and have greater physico-chemical stability than soybean lecithin.

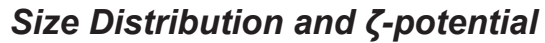 of Curcumin Liposomes}

The results of particle size distribution of curcumin liposomes are displayed in Figures 3A and 3B. The curcumin liposomes prepared with MFGM phospholipids showed an average particle diameter of $212.3 \mathrm{~nm}$ with a polydispersity index (PI) value of 0.261 . The small particle size provides stability against gravitational forces (Solans et al., 2005). The mean particle size of curcumin liposomes prepared with soybean lecithin was determined to be $471.4 \mathrm{~nm}$ with a PI value of 0.499 . Meanwhile, PI is an indicator of distribution broadness of particle sizes. A narrow distribution is shown when PI value is less than 0.3 , whereas the size distribution is considered to be broad when PI value is over 0.3 (Zweers et al., 2003). The MFGM liposomes possessed a narrow distribution, but the size distribution of soybean liposomes was broad.

The $\zeta$-potential of curcumin liposomes prepared with MFGM phospholipids and soybean lecithin was -48.60 and $-7.63 \mathrm{mV}$, respectively (Figures $3 \mathrm{C}$ and $3 \mathrm{D}$ ). Curcumin encapsulation may cause significant changes in the liposome surface structure, resulting in the change in orientation of the phosphatidylcholine head groups at the surface of liposomes, thus contributing to the negative $\zeta$-potential measured. Conventionally, the absolute value of $\zeta$-potential above $30 \mathrm{mV}$ is considered a high potential, which can give rise to high electrostatic repulsion among liposome particles, improving the stability of liposome system (Fatouros and Antimisiaris, 2002).

Chemical and physical stability of liposomes are closely related, and changes in any one of the 2 will affect the other. Chemical conversion of lipids membrane might change the structure of the system and the interfacial properties, thus resulting in the occurrence of physical modifications, such as particle growth and a decrease in $\zeta$-potential. The large liposome particles approach each other due to the loss of electrostatic repulsion and form an aggregation (Freitas and Muller, 1998). The consequences on size control, particle growth, and $\zeta$-potential are important considerations in the preparation of liposomes. The physico-chemical stability of lipids membrane showed variations due to their different compositions and structures (Heurtault et al., 2003). In the present study, curcumin liposomes prepared with MFGM phospholipids appeared to have a smaller particle size and a higher absolute value of $\zeta$-potential than those of prepared with soybean lecithin, indicating that MFGM liposome dispersion was a more stable system comparing to the soybean liposome dispersion.

\section{In Vitro Release of Curcumin from Liposomes}

The in vitro release of curcumin from liposomes, determined by dialysis method, is shown in Figure 4 . It is clear that the release behavior of encapsulated curcumin in liposomes was better than that of free curcumin. Within $4 \mathrm{~h}$, the release rate of curcumin in 2 kinds of liposome dispersions increased relatively rapidly considering that some unencapsulated curcumin quickly penetrated out from the dialysis bag, but their release rates were much lower than that of free curcumin solution. From 4 to $72 \mathrm{~h}$, the curcumin encapsulated in MFGM liposomes released gradually with the extension of time benefiting from the protection of lipid membranes. In contrast, the soybean liposomes exhibited a much higher release rate, and the fastest one was the free curcumin solution. Chemical properties of lipids membrane can change the load and release capacity of liposomes, and a liposome system with a higher chemical stability has a stronger regulatory capacity on release (Heurtault et al., 2003). The result of 

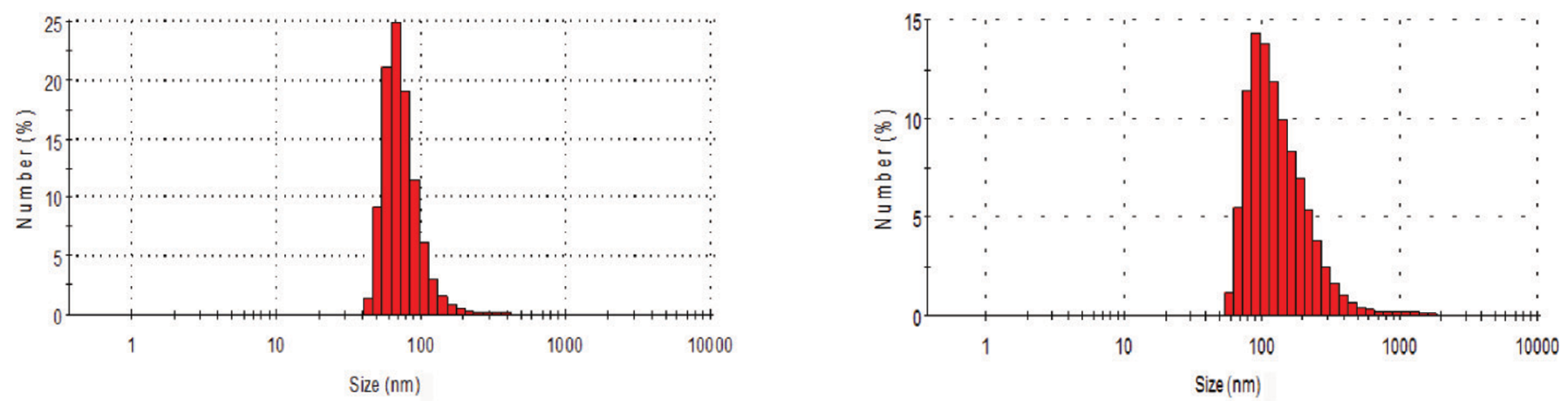

$\mathrm{C}$

Zeta Potential D istribution

$\mathrm{D}$

Zeta Potential Dis tribution
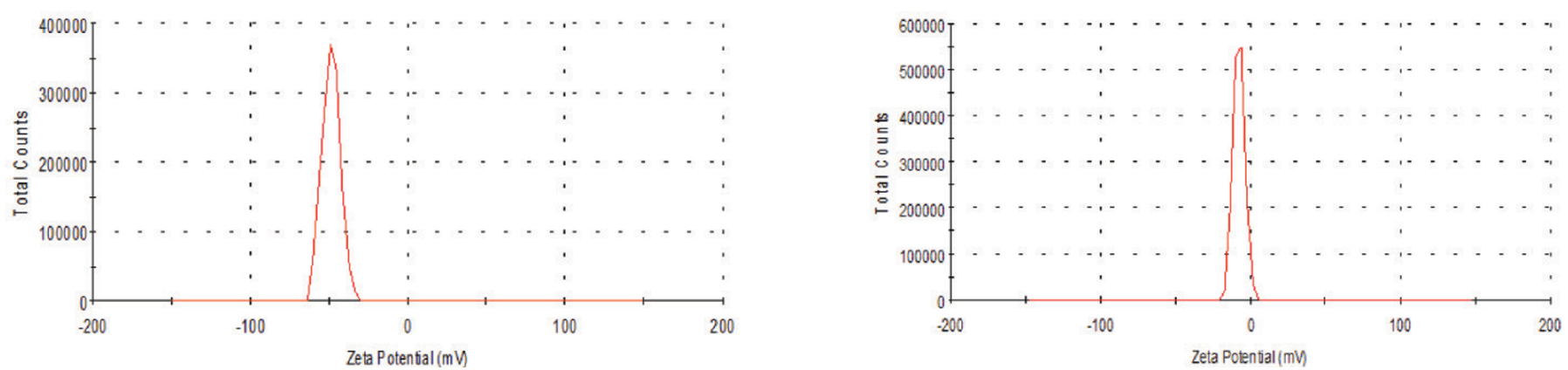

Figure 3. Size (diameter) distribution and $\zeta$-potential of curcumin liposomes. Size distribution of curcumin liposomes prepared with (A) milk fat globule membrane (MFGM) phospholipids or (B) soybean lecithin; $\zeta$-potential of curcumin liposomes prepared with (C) MFGM phospholipids or (D) soybean lecithin. Color version available online.

in vitro release also demonstrated curcumin liposomes prepared with MFGM phospholipids were superior to that prepared with soybean lecithin.

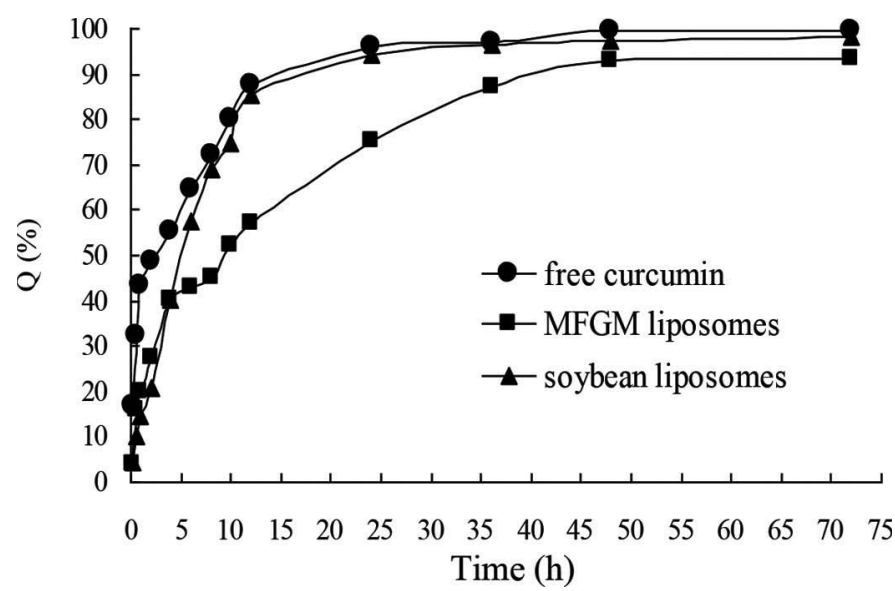

Figure 4. In vitro release profiles of curcumin liposomes. MGFM = milk fat globule membrane; $\mathrm{Q}=$ cumulative release rate.

\section{Effects of $p H$ on the Stability of Curcumin Liposomes}

The stability of curcumin liposomes prepared with MFGM phospholipids/soybean lecithin and free curcumin, subjected to different $\mathrm{pH}$ treatment, was displayed in Figure 5. Color change was visible in the free curcumin solution when the $\mathrm{pH}$ value range was 7.5 to 9 , indicating that curcumin was easy to degrade under alkaline condition. The high stability of curcumin under acidic condition might be contributed by the conjugated diene structure; however, when the $\mathrm{pH}$ was adjusted over 7.0, curcumin was degraded rapidly because of the destruction of the conjugated diene structure (Jovanovic et al., 1999). Unencapsulated curcumin was completely degraded after $40 \mathrm{~h}$ under alkaline condition, whereas the degradation of liposomal curcumin was relatively slower.

\section{Effects of Metal lons on the Stability of Curcumin Liposomes}

The changes in RR of curcumin caused by metal ions treatment are presented in Table 1 . The result shows no 


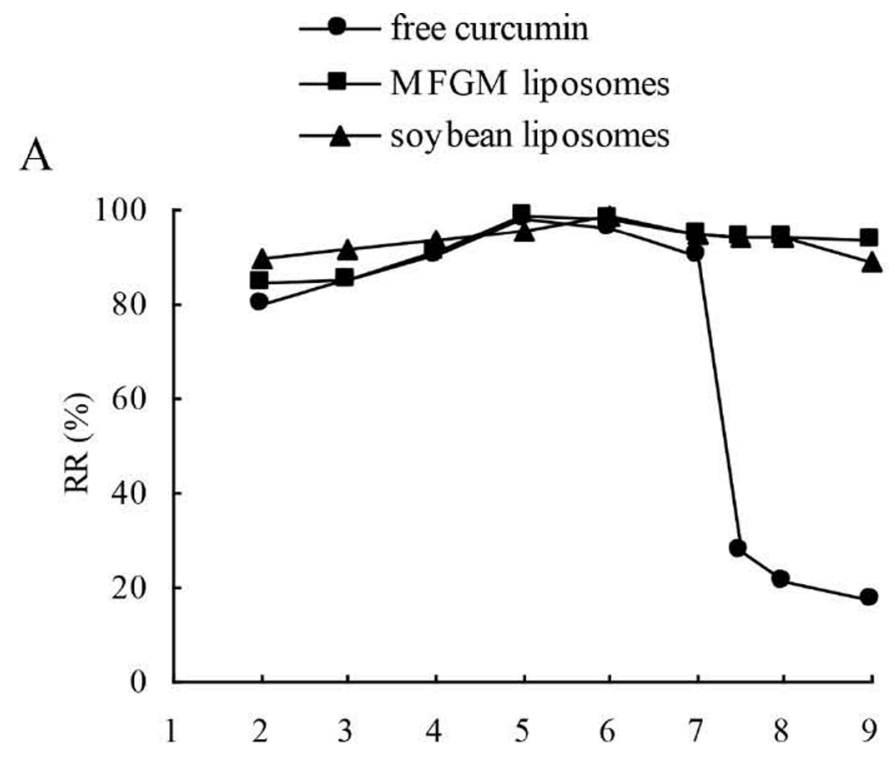

B

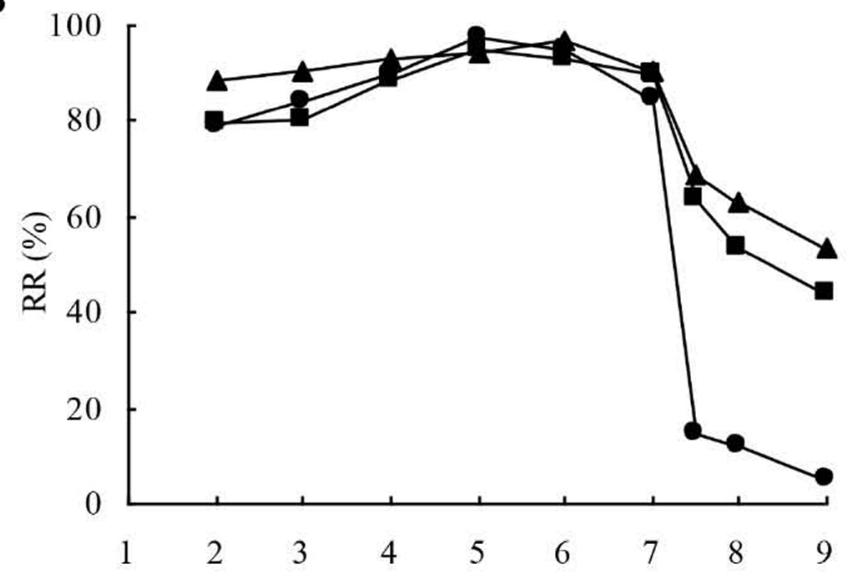

$\mathrm{C}$

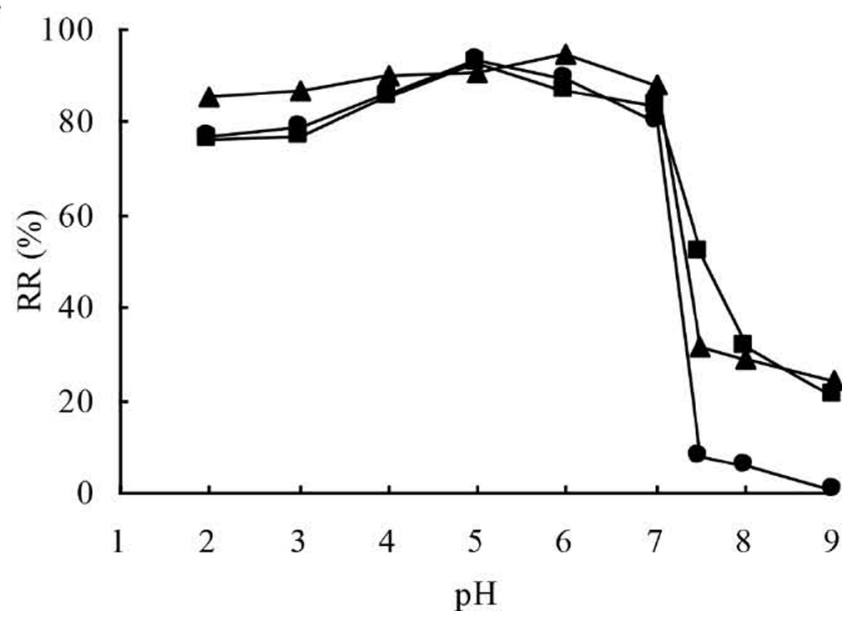

Figure 5. Effects of $\mathrm{pH}$ on the retention rate $(\mathrm{RR})$ of curcumin after (A) $30 \mathrm{~min}$, (B) $20 \mathrm{~h}$, and (C) $40 \mathrm{~h}$. obvious effect of metal ions on the degradation of curcumin, except $\mathrm{Fe}^{3+}$. A complete loss of free curcumin occurred under the $\mathrm{Fe}^{3+}$ condition, whereas the original encapsulated curcumin in liposomes was still retained. The RR of liposomes prepared with MFGM phospholipids was higher than that of prepared with soybean lecithin.

\section{Effects of Storage Conditions on the Stability of Curcumin Liposomes}

Stability determining factors are also storage conditions such as light, temperature, and oxygen. The storage stability of curcumin liposome dispersions was studied as a function of light, temperature, oxygen, and relative humidity. The MFGM liposomes, soybean liposomes, and free curcumin solution were prepared and stored under these conditions, respectively. The RR of curcumin in each sample was detected, and Figure 6 illustrates the effects of 4 storage conditions on the RR of curcumin.

Overall, lower losses of curcumin were observed during storage in the dark compared with under light (Figure 6A). After storage under light for $30 \mathrm{~d}$, the RR of curcumin in the liposome samples was around $70 \%$, whereas the RR of free curcumin was about $57 \%$. It seems clear that the liposomal core protected curcumin from the influence of light, resulting in a slow degradation of curcumin.

Figure $6 \mathrm{~B}$ shows that among the 3 temperature treatments, the highest loss of curcumin was incurred at the temperature of $50^{\circ} \mathrm{C}$ under dark conditions. The $\mathrm{RR}$ of curcumin was higher during storage at $4^{\circ} \mathrm{C}$ than $25^{\circ} \mathrm{C}$. It was reported that increasing temperature accelerates the hydrolysis of phospholipids, and the relationship between the hydrolysis rate constant and the temperature fitted the Arrhenius equation (Grit et al., 1993a). The permeability of the liposome membranes increases with the raise of temperature, leading to the leakage of curcumin from liposome interior and the degradation of curcumin. At low temperatures, the lipid membrane was in a colloidal state, the hydrolysis reaction of which requires higher activation energy (Grit et al., 1989). Generally, lower loss of curcumin occurred during storage in a refrigerator compared with at room temperature. The RR of liposomal curcumin decreased slowly with the increase of temperature, compared with that of the free curcumin.

As demonstrated in Figure 6C, in the presence of oxygen, the RR of curcumin decreased rapidly because the rate of oxidation is known to increase with oxygen. It is evident that the loss of encapsulated curcumin is markedly smaller than that of unencapsulated one. The results revealed that the encapsulation of curcumin 
A

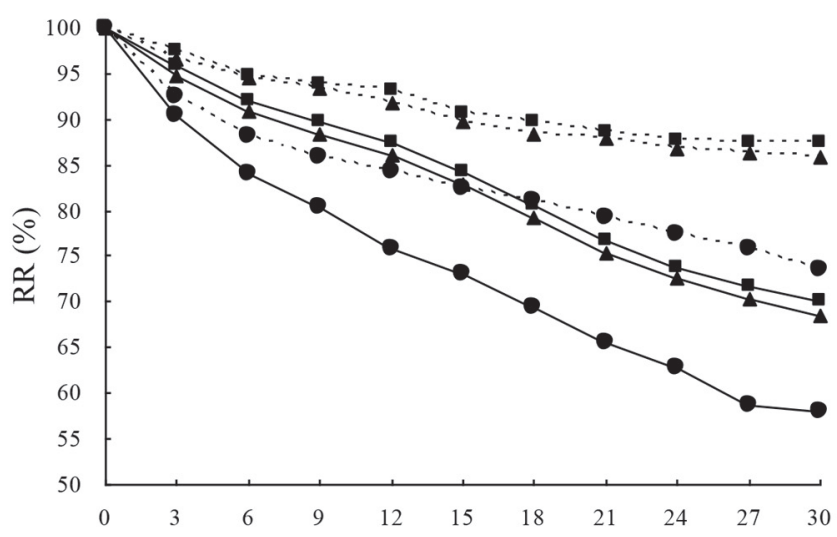

C

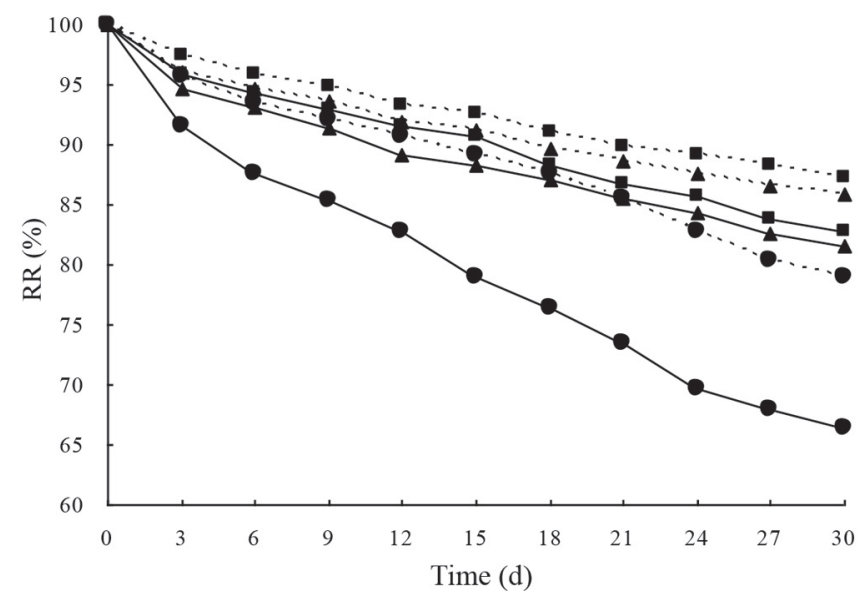

B
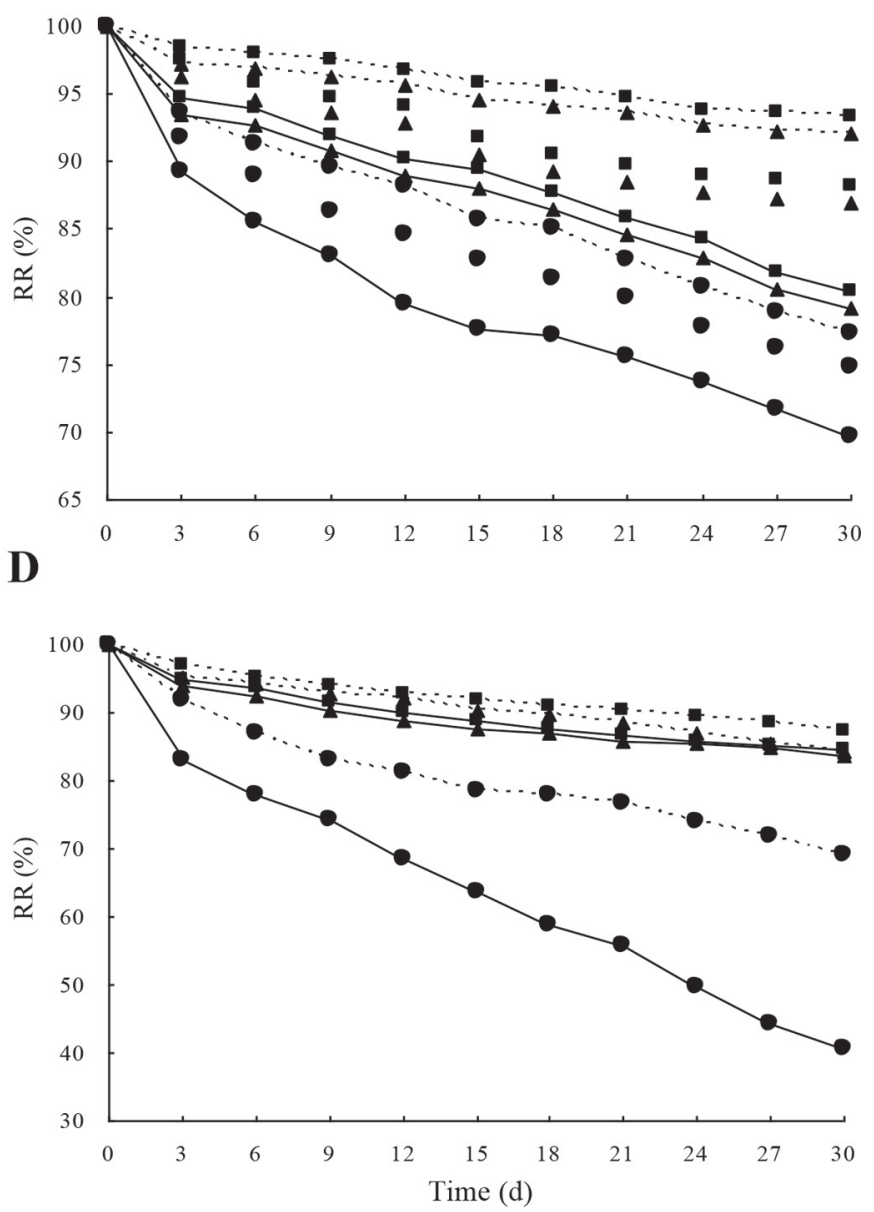

Figure 6. Effects of storage conditions on the retention rate $(\mathrm{RR})$ of curcumin. (A) Light (- - - in the dark; - under light); (B) temperature $\left(---4^{\circ} \mathrm{C}\right.$; no lines, $\left.25^{\circ} \mathrm{C} ;-50^{\circ} \mathrm{C}\right) ;(\mathrm{C})$ oxygen $(---$ without oxygen; - with oxygen); (D) relative humidity $(---33 \%$; - 80\%) of free curcumin solution $(\bullet)$, curcumin liposomes prepared with either milk fat globule membrane phospholipids (ם), or soybean lecithin $(\mathbf{\Lambda})$.

into liposomes could effectively improve the stability of curcumin.

The changes in the RR of curcumin were monitored during storage at 2 relative humidities (33 and $80 \%$ ). The relative humidity had an important effect on the $\mathrm{RR}$ of curcumin, as the free curcumin stored at $80 \%$ relative humidity destabilized much faster than those stored at 33\% relative humidity (Figure 6D). However, the RR of liposomal curcumin just had a slight change, which was far higher than that of free curcumin, supporting the protective effect of liposomes.

Results demonstrated that light, high temperature, oxygen, and high relative humidity accelerated the degradation of curcumin. The RR of liposomal curcumin was significantly higher than that of free curcumin during storage, indicating liposomes could effectively protect curcumin from chemical degradation. It has been reported that the rate of hydrolysis of saturated

Table 1. Effects of metal ions on the retention rate $(\mathrm{RR}, \%)$ of curcumin

\begin{tabular}{|c|c|c|c|c|c|c|}
\hline Item & $\mathrm{Ca}^{2+}$ & $\mathrm{Mg}^{2+}$ & $\mathrm{Zn}^{2+}$ & $\mathrm{Cu}^{2+}$ & $\mathrm{Fe}^{3+}$ & $\mathrm{Na}^{+}$ \\
\hline MFGM $^{1}$ liposomes & $97.20 \pm 0.94$ & $97.45 \pm 0.86$ & $97.97 \pm 0.74$ & $96.70 \pm 0.90$ & $94.36 \pm 0.69$ & $98.79 \pm 0.49$ \\
\hline Soybean liposomes & $97.66 \pm 0.67$ & $98.02 \pm 0.97$ & $96.00 \pm 0.92$ & $96.25 \pm 0.56$ & $90.51 \pm 0.81$ & $98.35 \pm 0.53$ \\
\hline
\end{tabular}

${ }^{1} \mathrm{MFGM}=$ milk fat globule membrane. 
soybean lecithin was lower than that of natural soybean lecithin (Grit et al., 1993b). The MFGM phospholipids contain a high level of sphingomyelin and higher degree of saturation of the fatty acid chains than soybean lecithin. Sphingomyelins are much more resistant to hydrolysis than lecithin, and saturated fatty acids tend to be less susceptible to oxidation compared with unsaturated ones (Thompson et al., 2006). These may protect the MFGM liposomes from hydrolytic and oxidative destabilization. In our results, the RR of MFGM liposomal curcumin was just slightly higher than that of soybean liposomal curcumin. They were all basically stable for at least $30 \mathrm{~d}$. Under a longer period of storage, MFGM liposomes may show a more markedly stability than soybean liposomes.

\section{CONCLUSIONS}

Under optimal preparing conditions, the encapsulation efficiency of curcumin liposomes prepared with MFGM phospholipids and soybean lecithin was about 74 and $63 \%$, respectively. The MFGM liposomes showed an average particle size of $212.3 \mathrm{~nm}$ and a $\zeta$-potential of $-48.60 \mathrm{mV}$, whereas the average particle size and $\zeta$-potential of soybean liposomes were $471.4 \mathrm{~nm}$ and $-7.63 \mathrm{mV}$. The MFGM liposomes had a significantly smaller particle size, higher absolute value of $\zeta$-potential, and slower release rate than soybean liposomes. The stability of MFGM liposomes was slightly higher or almost the same compared with soybean liposomes under the $\mathrm{pH}, \mathrm{Fe}^{3+}$, light, oxygen, temperature, and relative humidity conditions. The MFGM phospholipids are concluded to have potential advantages in the manufacture of liposomes used in food systems.

\section{ACKNOWLEDGMENTS}

This project was supported by the National Natural Foundation of China (31171631 and 31571773), Guangdong Province Science and Technology Plan Project (2011B031200005), and Guangdong Provincial Bureau of Ocean and Fishery Science and Technology (A201301C04).

\section{REFERENCES}

Anand, P., A. B. Kunnumakkara, R. A. Newman, and B. B. Aggarwal. 2007. Bioavailability of curcumin: Problems and promises. Mol. Pharm. 4:807-818.

Anand, P., S. G. Thomas, A. B. Kunnumakkara, C. Sundaram, K. B. Harikumar, B. Sung, S. T. Tharakan, K. Misra, I. K. Priyadarsini, K. N. Rajasekharan, and B. B. Aggarwal. 2008. Biological activities of curcumin and its analogues (Congeners) made by man and Mother Nature. Biochem. Pharmacol. 76:1590-1611.

Astaire, J. C., R. Ward, J. B. German, and R. Jimenez-Flores. 2003. Concentration of polar MFGM lipids from buttermilk by micro- filtration and supercritical fluid extraction. J. Dairy Sci. 86:22972307.

Began, G., E. Sudharshan, K. U. Sankar, and A. G. A. Rao. 1999. Interaction of curcumin with phosphatidylcholine: A spectrofluorometric study. J. Agric. Food Chem. 47:4992-4997.

Campagna, S., P. Cosette, G. Molle, and J.L. Gaillard. 2001. Evidence for membrane affinity of the C-terminal domain of bovine milk PP3 component. Biochim. Biophys. Acta 1513:217-222.

Crommelin, D. J. A. 1984. Influence of lipid composition and ionic strength on the physical stability of liposomes. J. Pharm. Sci. 73:1559-1563.

Dewettinck, K., R. Rombaut, N. Thienpont, T. T. Le, K. Messens, and J. Van Camp. 2008. Nutritional and technological aspects of milk fat globule membrane material. Int. Dairy J. 18:436-457.

Fatouros, D. G., and S. G. Antimisiaris. 2002. Effect of amphiphilic drugs on the stability and $\zeta$-potential of their liposome formulations: A study with prednisolone, diazepam, and griseofulvin. J. Colloid Interface Sci. 251:271-277.

Freitas, C., and R. H. Muller. 1998. Effect of light and temperature on zeta potential and physical stability in solid lipid nanoparticle (SLN (TM)) dispersions. Int. J. Pharm. 168:221-229.

Grit, M., and D. J. A. Crommelin. 1993. Chemical stability of liposomes: Implications for their physical stability. Chem. Phys. Lipids 64:3-18.

Grit, M., J. H. Smidt, A. Struijke, and D. J. A. Crommelin. 1989. Hydrolysis of phosphatidylcholine in aqueous liposome dispersions. Int. J. Pharm. 50:1-6.

Grit, M., W. J. M. Underberg, and D. J. A. Crommelin. 1993a. Hydrolysis of saturated soybean phosphatidylcholine in aqueous liposome dispersions. J. Pharm. Sci. 82:362-366.

Grit, M., N. J. Zuidam, W. J. Underberg, and D. J. Crommelin. 1993b. Hydrolysis of partially saturated egg phosphatidylcholine in aqueous liposome dispersions and the effect of cholesterol incorporation on hydrolysis kinetics. J. Pharm. Pharmacol. 45:490-495.

Heurtault, B., P. Saulnier, B. Pech, J. E. Proust, and J. P. Benoit. 2003. Physico-chemical stability of colloidal lipid particles. Biomaterials 24:4283-4300.

Jovanovic, S. V., S. Steenken, C. W. Boone, and M. G. Simic. 1999 $\mathrm{H}$-atom transfer is a preferred antioxidant mechanism of curcumin. J. Am. Chem. Soc. 121:9677-9681.

Keller, B. C. 2001. Liposomes in nutrition. Trends Food Sci. Technol. $12: 25-31$

Khar, A., A. M. Ali, B. V. V. Pardhasaradhi, Z. Begum, and R. Anjum. 1999. Antitumor activity of curcumin is mediated through the induction of apoptosis in AK-5 tumor cells. FEBS Lett. 445:165-168.

Kim, S. H., L. Haimovich-Caspi, L. Omer, Y. Talmon, and E. I. Franses. 2007. Effect of sonication and freezing-thawing on the aggregate size and dynamic surface tension of aqueous DPPC dispersions. J. Colloid Interface Sci. 311:217-227.

Kima, H. H. Y., and I. C. Baianua. 1991. Novel liposome microencapsulation techniques for food applications. Trends Food Sci. Technol. 2:55-61.

Lu, Q., D. C. Li, and J. G. Jiang. 2011. Preparation of a tea polyphenol nanoliposome system and its physicochemical properties. J. Agric. Food Chem. 59:13004-13011.

Mather, I. H. A. 2000. Review and proposed nomenclature for major proteins of the milk fat globule membrane. J. Dairy Sci. 83:203247.

Neethirajan, S., and D. S. Jayas. 2011. Nanotechnology for the food and bioprocessing industries. Food Bioprocess Tech. 4:39-47.

Patra, D., D. Ahmadieh, and R. Aridi. 2013. Study on interaction of bile salts with curcumin and curcumin embedded in dipalmitoylsn-glycero-3-phosphocholine liposome. Colloids Surf. B Biointerfaces 110:296-304.

Rombaut, R., J. V. Camp, and K. Dewettinck. 2005. Analysis of phospho- and sphingolipids in dairy products by a new HPLC method. J. Dairy Sci. 88:482-488.

Solans, C., P. Izquierdo, J. Nolla, N. Azemar, and M. J. Garcia-Celma. 2005. Nano-emulsions. Curr. Opin. Colloid. 10:102-110.

Spitsberg, V. L. 2005. Bovine milk fat globule membrane as a potential nutraceutical. J. Dairy Sci. 88:2289-2294. 
Takahashi, M., S. Uechi, K. Takara, Y. Asikin, and K. Wada. 2009. Evaluation of an oral carrier system in rats: Bioavailability and antioxidant properties of liposome-encapsulated curcumin. J. Agric. Food Chem. 57:9141-9146.

Taylor, T. M., P. M. Davidson, B. D. Bruce, and J. Weiss. 2005. Liposomal nanocapsules in food science and agriculture. Crit. Rev. Food Sci. Nutr. 45:587-605.

Thompson, A. K., D. Haisman, and H. Singh. 2006. Physical stability of liposomes prepared from milk fat globule membrane and soya phospholipids. J. Agric. Food Chem. 54:6390-6397.

Thompson, A. K., M. R. Mozafari, and H. Singh. 2007. The properties of liposomes produced from milk fat globule membrane material using different techniques. Lait 87:4-5.

Thompson, A. K., and H. Singh. 2006. Preparation of liposomes from milk fat globule membrane phospholipids using a Microfluidizer. J. Dairy Sci. 89:410-419.

Vermette, P., S. Taylor, D. Dunstan, and L. Meagher. 2002. Control over PEGylated-liposome aggregation by NeutrAvidin-biotin in- teractions investigated by photon correlation spectroscopy. Langmuir 18:505-511

Xia, F., D. D. Hu, H. Y. Jin, Y. P. Zhao, and J. M. Liang. 2012. Preparation of lutein proliposomes by supercritical anti-solvent technique. Food Hydrocoll. 26:456-463.

Ye, A., H. Singh, M. W. Taylor, and S. Anema. 2002. Characterization of protein components of natural and heat-treated milk fat globule membranes. Int. Dairy J. 12:393-402.

Yotsuyanagi, T., H. Hashimoto, M. Iwata, and K. Ikeda. 1987. Effect of cholesterol on liposome stability to ultrasonic disintegration and sodium cholate solubilization. Chem. Pharm. Bull. (Tokyo) 35:1228-1233.

Zweers, M. L. T., D. W. Grijpma, G. H. M. Engbers, and J. Feijen. 2003. The preparation of monodisperse biodegradable polyester nanoparticles with a controlled size. J. Biomed. Mater. Res. B. Appl. Biomater. 66:559-566. 